\title{
Stromal cell induction of regulatory dendritic cells
}

\author{
Benjamin M. J. Owens ${ }^{\dagger}$ and Paul M. Kaye* \\ Centre for Immunology and Infection, Hull York Medical School and Department of Biology, University of York, York, UK
}

\section{Edited by:}

Burkhard Ludewig, Kantonal Hospital St. Gallen, Switzerland

\section{Reviewed by:}

Scott Mueller, The University of

Melbourne, Australia

Nicola Laraine Harris, École

Polytechnique Fédérale de Lausanne

Switzerland

\section{*Correspondence}

Paul M. Kaye, Centre for Immunology and Infection, Hull York Medical

School and Department of Biology,

University of York, Wentworth Way,

York YO10 5DD, UK.

e-mail:paul.kaye@york.ac.uk

\section{${ }^{+}$Current address:}

Benjamin M. J. Owens, Translational

Gastroenterology Unit, Nuffield

Department of Medicine,

Experimental Medicine Division,

University of Oxford, Oxford, UK.
Dendritic cells (DCs) are specialized antigen presenting cells of bone marrow origin that can exist in tissues in either an immature or mature state. DCs have a myriad of roles in immunity and tolerance induction, but are perhaps best known for their role in the activation and differentiation of naïve $T$ cells at the onset of an acquired immune response. Over the past decade, a body of literature has developed that suggests that DCs, as well as many other myeloid cell populations, are also capable of exerting "regulatory" effects on T cell responses. However, relatively little is known regarding the mechanisms by which such regulatory myeloid cells arise in vivo. In this mini-review, we first define the characteristics of "regulatory" DCs (rDCs) and then focus on the contribution of non-hematopoietic stromal cells to their generation within specific tissue microenvironments. We also highlight areas of research that warrant future attention, arguing for a focusing of efforts toward a better understanding of the features of stromal cell populations that enable the induction of rDCs. Finally, we discuss how an understanding of stromal cell-myeloid cell interactions may lead to new therapeutic strategies for cancer, autoimmunity, and infectious disease.

Keywords: stromal cells, dendritic cells, Immune regulation, IL-10, inflammation, infection

\section{INTRODUCTION}

Dendritic cells (DCs) lie at the interface of innate and adaptive immunity, playing a critical role in the initiation of effective $\mathrm{T}$ cell-mediated immune responses. Paradoxically, DCs also have the potential to exert powerful negative regulatory effects on the immune system (Steinman et al., 2003). This potential for dampening immunity has spawned great interest in the context of cellbased therapeutic intervention in a variety of autoimmune and inflammatory contexts (Kalantari et al., 2011). Alongside several populations of conventional CD11 $\mathrm{c}^{\text {hi }} \mathrm{DCs}(\mathrm{cDCs})$, there are many other myeloid cell populations capable of antigen presentation and exerting both positive and negative effects on $\mathrm{T}$ cell responses. This has given rise to a literature that contains a significant level of confusion. That many of these myeloid cells also share phenotypic characteristics has only compounded the problem. For example, the assignment of cells as DCs based solely on CD11c expression has not always been helpful (Drutman et al., 2012). The relationship between $\mathrm{cDCs}$ and other myeloid cells is further complicated by the existence of convergent differentiation pathways, particularly under inflammatory conditions (Geissmann et al., 2010). Nevertheless, recent data strongly support the concept that cDCs belong to a distinct immune cell lineage (Meredith et al., 2012; Satpathy et al., 2012). The identification of key lineage-related transcription factors should allow rapid progress in determining the relationships between cDCs and other myeloid cell populations and add some clarity to studies of the regulatory properties of these cells.

Whilst the regulatory potential of cDCs in vivo has long been appreciated (Hawiger et al., 2001), consensus regarding the phenotypic features of $\mathrm{cDCs}$ and myeloid cells with regulatory properties has been hard to reach. Historically, "immature" cDCs were classed as those that had yet to receive a pathogen-derived signal and existed in the tissues in a state of readiness for antigen presentation, with high levels of endocytosis to facilitate antigen capture and large intracellular pools of MHCII. Upon pathogen recognition by TLRs or other pattern recognition receptors, cDCs "mature" and in so doing, shut down endocytosis in favor of MHCII-peptide display, heightened expression of co-stimulatory molecules, and the secretion of cytokines that direct naïve $\mathrm{T}$ cell differentiation. Early literature suggested that immature cDCs may also be endowed with regulatory function, although this may represent an oversimplification (Kleindienst et al., 2005). Conversely, all subsets of splenic $\mathrm{CDC}$ have recently been shown to be capable of producing the regulatory cytokine IL-10, even after TLR induced maturation (Maroof and Kaye, 2008; Owens et al., 2012). In the context of chronic Leishmania donovani infection, IL-10-producing cDCs are capable of antigen presentation and the induction of naïve $\mathrm{T}$ cell proliferation in vitro (Owens et al., 2012), making them functionally distinct from rDCs as we define below. Against this background, where pleiotropic function characterizes cDCs, it becomes pertinent to ask whether there are distinct populations of DCs (regulatory DCs; rDCs) in which regulatory function is hardwired, and how stromal cell populations can contribute to their generation (Figure 1). The remainder of this review will focus on addressing this question.

\section{REGULATORY DCS: CHARACTERIZATION AND FUNCTION}

Amongst cytokines, IL-10 has become synonymous with the concept of regulation, yet as discussed above cDCs under appropriate circumstances are quite capable of producing this cytokine. Hence, 




FIGURE 1 | Relationships between rDCs and cDCs. cDCs are known to originate from a hematopoietic stem cell precursor, through a sequence of events (not shown) that culminates in production of tissue precursor cells (pre-cDCs). In tissue, cDCs exist as immature cells, but functionally mature in response to microbial sensing mediated through various pattern recognition receptors (PRRs). Mature $\mathrm{cDCs}$ have an enhanced capacity to prime naive T cells and induce their differentiation (shown here for Th1 cells). Mature cDCs may also help terminate excessive T cell responses by inducing a "self regulatory" phenotype, e.g., through the induction of IL-10 production by Th1 cells. Under steady state conditions, stromal cells (blue) are able to induce the generation of $\mathrm{IL}-10$ and $\mathrm{PGE}_{2}$-producing rDCs from c-kit ${ }^{+}$progenitors by production of $\mathrm{PGE}_{2}, \mathrm{IL}-6$, and IL-10, as well as direct interactions mediated by Fibronectin. During the chronic inflammation associated with L. donovani infection, IL-10-producing $r D C$ generation is enhanced by the production of CCL8 and CXCL12 by splenic stromal cells that in many cases are directly parasitized (red). The stage(s) of development at which $\mathrm{cDCs}$ or their precursors are able to differentiate into regulatory DCs is not yet defined. Functionally, regulatory DCs are best defined by their capacity to inhibit the proliferative capacity of T cells. rDCs employ multiple mechanisms to suppress naïve T cell proliferation, including the direct production of $\mathrm{IL}-10$ and the induction of FoxP3 ${ }^{+} \mathrm{nTreg}$ and $\mathrm{IL}-10^{+}$ Tr1 Treg populations.
IL-10 alone could not be a sufficient criterion by which to distinguish rDCs. Although there is evidence of a rDC population with functions that are distinct from cDCs, there is currently nothing known as to the extent of plasticity within this group of myeloid cells. In particular it is not yet clear whether $\mathrm{rDCs}$ represent a terminally differentiated DC phenotype, or a transient functional state reflecting phenotypic changes of myeloid cells in distinct tissue microenvironments. Despite such ambiguity in the nature of rDCs, some of the strongest evidence in support of the existence of this population has come from the study of how fibroblasts and endothelial cells impact on DC development from hematopoietic stem cells or committed myeloid progenitors.

Stromal cell induction of rDC differentiation can occur in multiple tissues even in the absence of pathogen recognition and inflammation, suggesting that this is a normal homeostatic process. To date, stromal cell-induced rDCs have been reported in murine spleen (Svensson et al., 2004; Zhang et al., 2004; Tang et al., 2006; Nguyen Hoang et al., 2010; Xu et al., 2012), liver (Xia et al., 2008), kidney (Huang et al., 2009), lung (Li et al., 2008), and tumor tissue (Liu et al., 2009). Despite their divergent tissue localization, the majority of studies reporting stromal cell-induced $\mathrm{rDCs}$ have characterized them as populations of CD11 $\mathrm{c}^{\mathrm{lo}} \mathrm{MHCII} \mathrm{I}^{\mathrm{lo} \text { int }}$ $\mathrm{CD}_{11} \mathrm{~b}^{+}$cells, based on surface protein expression assessed by flow cytometry. Splenic rDCs have also been reported to express CD45RB (Wakkach et al., 2003; Svensson et al., 2004), although the functional significance of this is not known. Expression of co-stimulatory molecules such as CD40, CD80, and CD86 is generally lower on rDCs than cDCs, suggesting an impaired capacity to deliver activatory signals to naïve $\mathrm{T}$ cell populations, although high co-stimulatory molecule expression (particularly CD80) by rDCs has been reported in some contexts (reviewed by Svensson and Kaye, 2006). 
Several other characteristics of stromal cell-induced rDCs allow for their more rigorous identification. Multiple studies have reported that $\mathrm{rDCs}$ are major producers of the anti-inflammatory cytokine IL-10, including those from spleen (Svensson et al., 2004; Zhang et al., 2004; Tang et al., 2006), liver (Xia et al., 2008), kidney (Huang et al., 2009), and lung (Li et al., 2008), suggesting that IL-10 production is a conserved feature of rDCs, irrespective of tissue localization, or origin. This preferential IL-10 production can be driven by TLR triggering, and at least partially relies on ERK signaling (Qian et al., 2006). However, conventional CD11 $\mathrm{c}^{\text {hi }} \mathrm{MHCII}{ }^{\mathrm{hi}} \mathrm{CDC}$ are also capable of abundant IL-10 production (Saraiva and O'Garra, 2010), a process particularly pronounced during chronic parasitic infection (Maroof and Kaye, 2008; Owens et al., 2012) indicating that IL-10 production is not unique to rDCs.

Distinctive functional properties of rDCs provide perhaps the best method for their identification, as rDCs utilize multiple pathways in order to exert their immune regulatory function. Several reports suggest that $\mathrm{rDC}$ are capable of modulating $\mathrm{T}$ cell proliferation in vitro and in vivo, with splenic stromal-induced rDCs suppressing proliferation via IL-10, TGF $\beta$, and/or nitric oxide (NO) production (Svensson et al., 2004; Zhang et al., 2004; Tang et al., 2006), and pulmonary stromal-induced rDCs inhibiting $\mathrm{T}$ cell proliferation via production of Prostaglandin- $\mathrm{E}_{2}\left(\mathrm{PGE}_{2} ; \mathrm{Li}\right.$ et al., 2008). Importantly this capacity for stromal-induced rDCs to suppress $\mathrm{T}$ cell proliferation does not appear to be dependent on the presence of stromal cells within the assays, as stromal-induced rDCs are capable of suppressing $\mathrm{T}$ cell proliferation during in vitro co-culture solely with T cells, as well as after adoptive transfer in vivo. This therefore indicates a regulatory process distinct from that recently reported for lymph node (LN) fibroblastic reticular cells (FRCs), whereby nitric oxide produced by LN FRCs regulates $\mathrm{T}$ cell proliferation via direct effects on $\mathrm{T}$ cells, in addition to modulating cDC function (Khan et al., 2011; Lukacs-Kornek et al., 2011; Siegert et al., 2011). Stroma-induced rDCs within tumors are able to utilize an alternative mechanism involving the direct production of Arginase-1 (Liu et al., 2009). This results in the metabolism of L-Arginine and consequent suppression of $\mathrm{T}$ cell proliferation in vitro and in vivo. In contrast, splenic stromainduced rDCs specifically recruit $\mathrm{CXCR}^{+}$Th1 cells to more efficiently suppress their proliferation, via the IFN $\alpha / \beta$-dependent production of IP-10 after TLR triggering (Qian et al., 2007). More recent evidence has suggested that splenic $\mathrm{rDCs}$ are also able to directly induce apoptosis of activated $\mathrm{CD} 4^{+} \mathrm{T}$ cells by a process involving NO, Fas-Ligand, and IFN $\gamma$ (Xu et al., 2012), a mechanism also reported to occur in the liver (Xia et al., 2008). This capacity for direct suppression of $\mathrm{CD} 4{ }^{+} \mathrm{T}$ cell proliferation, even after TLR induced "maturation," is a cardinal feature of rDCs that allows for them to be distinguished from cDCs.

In addition to their direct modulatory effects on activated $\mathrm{T}$ cells, rDCs can also employ indirect immune regulatory mechanisms, in many cases by the induction of specialized populations of regulatory T cells (Treg). Splenic stroma-induced rDCs directly induce IL-10-producing Tr1 Treg in vitro (Svensson et al., 2004), whereas rDCs induced by pulmonary stromal cells can induce populations of Foxp $3^{+}$natural Treg (Li et al., 2008). Whether the differential induction of Treg populations from rDCs induced by stromal cells of distinct tissues reflects the polarization of rDC subsets is currently unclear. It will be important to define stromainduced $\mathrm{rDCs}$ from distinct tissues in more detail to elucidate whether further functional subsets exist, in addition to the cues that drive their differentiation. However, Treg induction by rDCs does not always contribute to their regulatory potential (Tang et al., 2006) and indeed rDCs have been reported to activate NK cells in some circumstances (Qian et al., 2006). These divergent functions suggest that although rDCs are capable of suppressing the cellular components of an active immune response, in different contexts rDCs may also act in an immune stimulatory fashion.

Taking these multiple parameters into consideration, a "minimal" definition of stromal-induced $\mathrm{rDCs}$ can be reached as CD11 $\mathrm{c}^{\text {lo/int }} \mathrm{MHCII}^{\mathrm{lo} / \text { int }} \mathrm{CD} 1 \mathrm{~b}^{+}$myeloid cells, capable of IL-10 production, the direct suppression of $\mathrm{CD}^{+}{ }^{+} \mathrm{T}$ cell proliferation and (in some cases) the induction of Treg. Although several other functional features can also contribute to the identification or characterization of rDCs, these are either tissue-context dependent, or have not yet been assessed in all stromal-induced rDC populations.

\section{SPECIFIC CONTEXTS LEADING TO THE INDUCTION OF rDCs BY STROMAL CELLS}

It is clear that in several tissue contexts stromal cells can induce rDCs, but direct evidence as to whether this process is regulated by infection or inflammation is more limited. One exception is experimental visceral leishmaniasis (EVL), a chronic infection caused by the intracellular parasite L. donovani (Kaye et al., 2004). Splenic stromal cells from mice infected with $L$. donovani have an enhanced capacity to direct hematopoietic progenitors toward a rDC phenotype in vitro (Svensson et al., 2004), a process at least in part dependent upon infection-modulated levels of the chemokine CCL8 (Nguyen Hoang et al., 2010). The precise mechanisms by which infection itself enhances the capacity for stromal cells to support rDC induction during chronic inflammation are not known, but as stromal cells are targets of Leishmania infection (Bogdan et al., 2000) it is feasible that direct parasite modulation of stromal cell function may represent a strategy for manipulating host defense mechanisms in favor of the invading pathogen (Svensson and Kaye, 2006). Whether similar alterations in stromal cells occur during other parasitic infections with an abundance of CD11 $c^{\text {lo }}$ rDCs (Li et al., 2011; Smith et al., 2011), and whether rDCs are associated with chronic infection by viral or bacterial pathogens will require further investigation.

As stromal cells from organs considered mucosal (Li et al., 2008; Huang et al., 2009) or with specialized properties related to tolerance induction (Xia et al., 2008) have been reported to induce rDCs, it is possible that this is a generalized feature of stromal populations from these sites. It is not yet known whether stromal cells from other mucosal organs, such as the skin or intestine, are specialized for the induction of rDCs.

\section{MECHANISMS OF rDC INDUCTION BY STROMAL CELLS}

Unlike the relatively conserved phenotypic and functional characteristics of rDCs, there are multiple reported mechanisms by which stromal cells induce these cells. Physical contact between splenic stromal cells and mature cDCs has been shown to be required 
for their polarization toward a rDC phenotype, in a process also dependent on fibronectin (Zhang et al., 2004). However, kidney stromal cells can induce rDCs by a process that does not require cell-cell contact (Huang et al., 2009), indicating heterogeneity in the mechanisms underlying stromal cell-induced rDC differentiation. This is perhaps not surprising given the heterogeneity in stromal cell populations themselves, as shown clearly by recent transcriptional analysis of stromal cell subsets within lymphoid tissue (Malhotra et al., 2012).

Diverse stromal cell-derived products such as IL-6 (Huang et al., 2009), CCL8 and CXCL12 (Nguyen Hoang et al., 2010), TGF 3 (Li et al., 2008), and M-CSF (Xia et al., 2008) have been reported to impact upon the stromal cell induction of rDCs, but it is unclear as to whether redundancy exists in this system. In addition to rDCs being producers of $\mathrm{PGE}_{2}$ and IL-10, the release of these mediators by stromal cells has also been implicated in their generation, with splenic endothelial-produced IL-10 (Tang et al., 2006) and tumor stroma-derived $\mathrm{PGE}_{2}$ (Liu et al., 2009) playing a role in the induction of $\mathrm{rDC}$ populations.

A potent immunoregulatory circuit endowing human DCs with IL-27-dependent regulatory potential was recently described (Ilarregui et al., 2009), relying on the carbohydrate-binding protein Galectin-1. As Galectin-1 is expressed by stromal cells (Jung et al., 2007), this raises the intriguing possibility that stromal populations are also capable of delivering signals that skew conventional human DCs toward regulatory capacity, in addition to the differentiation of specific rDC populations.

\section{FUTURE QUESTIONS}

As evidenced by this short review, there is still much to be revealed regarding the precise mechanisms by which stromal cells induce the differentiation and/or expansion of rDCs (Figure 1). Indeed there are large gaps in our knowledge regarding the precise identity of rDCs, whether subsets of these cells exist in vivo and the lineage relationship of rDCs to conventional DC populations. Transcriptional and epigenetic profiling of rDCs from a multitude of tissue sites and disease states will allow for these questions to begin to be addressed. As many of the mediators previously reported to be important for rDC generation are produced widely within the immune system, it is important to determine how they contribute to the generation of rDCs within a defined tissue microenvironment. As it is likely that a multitude of synergistic signals underlie the induction of rDCs by stromal cells, revealing the extent of redundancy in this system will be key in finding pathways essential for rDC induction by stromal cells. In addition, extending our knowledge of the processes by which pathogens, vaccination, or chronic inflammation can modulate stromal cell function, and thus favor or suppress $\mathrm{rDC}$ induction in vivo will be crucial when considering therapeutic strategies aimed at manipulating rDC abundance or function.

Furthermore, a more detailed analysis of the stromal microenvironment that supports $\mathrm{rDC}$ generation in vitro will provide clues as to novel mechanisms responsible for their in vivo induction. This type of analysis has already been performed for splenic stromal cell populations capable of inducing immature DC populations in vitro (Despars et al., 2008), identifying gene signatures associated with this functional capacity. Extending this approach to rDC induction will likely reveal much useful data in this area.

More fundamentally, it is essential that we gain a much deeper understanding of the stromal cell populations capable of rDC induction in vivo. Even within the same organ it would appear that diverse stromal populations such as fibroblasts (Svensson et al., 2004) and endothelial cells (Tang et al., 2006) are capable of rDC induction. Clarity on whether distinct differences in stromal cells both within and between organs results in a differing capacity for rDC induction, or alternative mechanisms by which induction occurs, will likely reveal much about the biological processes required for stromal cells to induce rDC populations. Key to this may be identification of the tissue specific niches for rDC development (as this will define the local stromal cell population) coupled to transcriptional and/or proteomic analysis of the stromal cells at such sites. In particular the application of advanced imaging techniques such as intravital microscopy or whole mount histology could be applied to identifying stromal niches for rDC generation in situ, allowing for the visualization of the distinct microenvironments that facilitate the induction of these cells. However these approaches will necessitate the development of specific tools for the identification of rDCs in vivo. Such experiments are also likely to challenge the conclusions drawn from conventional experimental approaches.

To bring clarity to the field, the following approaches should therefore be taken to address the major outstanding research questions regarding stromal cell-induced rDCs:

- Transcriptional and epigenetic analysis of stromal-induced rDCs from distinct tissues to determine whether subsets exist and their relationship to cDCs.

- Imaging approaches to visualize the stromal cell-rDC niche in vivo, which will require the development of new tools for rDC identification.

- Transcriptional and epigenetic analysis of stromal cells capable of rDC induction, specifically comparing these profiles to those of stromal cells from distinct tissues and/or disease states.

Extending our knowledge of both rDCs and the stromal cells that induce them may allow for the potential therapeutic benefits of this immunoregulatory axis to be realized.

\section{HARNESSING STROMAL CELL NICHES FOR THERAPEUTIC IMMUNE REGULATION}

With patient-specific pro-inflammatory DC infusions effective in phase III trials for prostate cancer (Kantoff et al., 2010), the use of myeloid cell populations to modulate immune responses in humans is rapidly becoming a clinical reality. Applying this rationale to the design of alternative therapeutic myeloid cell infusions aimed instead at repressing immune responses is already under close scrutiny (Kalantari et al., 2011; Lutz, 2012), with progress in this area likely soon. As stromal cell-induced rDCs are potent negative regulators of inflammation and improve outcome in preclinical models of hepatic and pulmonary insult (Li et al., 2008; Xia et al., 2008), such cell populations - once characterized fully in humans - would provide a promising candidate for therapeutic infusion. 
Although clearly feasible, the ex vivo expansion of myeloid cell populations for infusion has disadvantages. Instead, harnessing the capacity for stromal cells to regulate rDC induction by specifically targeting them for functional modulation in vivo could provide a method to enhance $\mathrm{rDC}$ induction for the amelioration of autoimmune or inflammatory disease, or conversely repress rDC induction during the immunosuppression associated with chronic infection and cancer. Stromal targeting approaches have already attracted much interest in the oncology field (Engels et al., 2012). Experimental therapeutics have included the targeting of potent cytotoxic agents to tumor stroma by conjugation to a collagen IV-specific monoclonal antibody (Yasunaga et al., 2011), specific enzymatic degradation of tumor stroma to enhance stromal remodeling (Provenzano et al., 2012), and therapeutic ablation of cancer stromal cells in

\section{REFERENCES}

Bogdan, C., Donhauser, N., Döring, R., Röllinghoff, M., Diefenbach, A., and Rittig, M. G. (2000). Fibroblasts as host cells in latent leishmaniasis. $J$. Exp. Med. 191, 2121-2130.

Despars, G., Periasamy, P., Tan, J., Abbey, J., O'Neill, T. J., and O'Neill, H. C. (2008). Gene signature of stromal cells which support dendritic cell development. Stem Cells Dev. 17, 917-927.

Drutman, S. B., Kendall, J. C., and Trombetta, E. S. (2012). Inflammatory spleen monocytes can upregulate CD11c expression without converting into dendritic cells. J. Immunol. 188, 3603-3610.

Engels, B., Rowley, D. A., and Schreiber, H. (2012). Targeting stroma to treat cancers. Semin. Cancer Biol. 22, 41-49.

Geissmann, F., Manz, M. G., Jung, S., Sieweke, M. H., Merad, M., and Ley, K. (2010). Development of monocytes, macrophages, and dendritic cells. Science 327, 656-661.

Hawiger, D., Inaba, K., Dorsett, Y., Guo, M., Mahnke, K., Rivera, M., Ravetch, J. V., Steinman, R. M., and Nussenzweig, M. C. (2001). Dendritic cells induce peripheral $\mathrm{T}$ cell unresponsiveness under steady state conditions in vivo. J. Exp. Med. 194, 769-779.

Huang, Y., Johnston, P., Zhang, B., Zakari, A., Chowdhry, T., Smith, R. R., Marbán, E., Rabb, H., and Womer, K. L. (2009). Kidneyderived stromal cells modulate dendritic and T cell responses. J. Am. Soc. Nephrol. 20, 831-841.

Ilarregui, J. M., Croci, D. O., Bianco, G. A., Toscano, M. A., Salatino, M., Vermeulen, M. E., Geffner, J. R., and Rabinovich, G. A. (2009). Tolerogenic signals delivered by dendritic cells to $\mathrm{T}$ cells through a galectin-1-driven immunoregulatory circuit involving interleukin 27 and interleukin 10. Nat. Immunol. 10, 981-991.

Jung, E. J., Moon, H. G., Cho, B. I., Jeong, C. Y., Joo, Y. T., Lee, Y. J., Hong, S. C., Choi, S. K., Ha, W. S., Kim, J. W., Lee, C. W., Lee, J. S., and Park, S. T. (2007). Galectin-1 expression in cancer-associated stromal cells correlates tumor invasiveness and tumor progression in breast cancer. Int. J. Cancer 120, 2331-2338.

Kalantari, T., Kamali-Sarvestani, E., Ciric, B., Karimi, M. H., Kalantari, M., Faridar, A., Xu, H., and Rostami, A. (2011). Generation of immunogenic and tolerogenic clinical-grade dendritic cells. Immunol. Res. 51, 153-160.

Kantoff, P. W., Higano, C. S., Small, E. J., Whitmore, J. B., Frohlich, M. W., and Schellhammer, P. F. (2010). Sipuleucel-T immunotherapy for castration-resistant prostate cancer. N. Engl. J. Med. 363, 411-422.

Kaye, P. M., Svensson, M., Ato, M., Maroof, A., Polley, R., Stager, S., Zubairi, S., and Engwerda, C. R. (2004). The immunopathology of experimental visceral leishmaniasis. Immunol. Rev. 201, 239-253.

Khan, O., Headley, M., Gerard, A., Wei, W., Liu, L., Krummel, M. F. (2011). Regulation of T cell priming by lymphoid stroma. PLoS ONE 6, e26138. doi:10.1371/journal.pone.0026138

Kleindienst, P., Wiethe, C., Lutz, M. B., and Brocker, T. (2005). Simultaneous induction of CD4 $\mathrm{T}$ cell tolerance and CD8 T cell immunity by semimature dendritic cells. J. Immunol. 174, 3941-3947.

Kraman, M., Bambrough, P. J., Arnold, J. N., Roberts, E. W., Magiera, L., Jones, J. O., Gopinathan, A., Tuveson, D. A., and Fearon, D. T. (2010). Suppression of antitumor immunity by stromal cells expressing fibroblast activation protein-alpha. Science 330, 827-830.

murine tumor models (Kraman et al., 2010), but stromal targeting approaches are not usually considered in other therapeutic contexts.

By expanding our knowledge regarding the detailed mechanisms underlying stromal cell - regulatory dendritic cell interactions, we may advance one step closer to the ultimate goal of subtly manipulating immune function by targeting stromal cells within an inflammatory microenvironment.

\section{ACKNOWLEDGMENTS}

We thank Dr. Claire Pearson for critical review of the manuscript. Research in PMK's laboratory is funded by the UK Medical Research Council and the Wellcome Trust. Benjamin M. J. Owens was recipient of a MRC Doctoral Training Award and is currently supported by the Wellcome Trust.

Li, Q., Guo, Z., Xu, X., Xia, S., and Cao, X. (2008). Pulmonary stromal cells induce the generation of regulatory DC attenuating T-cell-mediated lung inflammation. Eur. J. Immunol. 38, 2751-2761.

Li, Z., Liu, G., Chen, Y., Liu, Y., Liu, B., and Su, Z. (2011). The phenotype and function of naturally existing regulatory dendritic cells in nematode-infected mice. Int. J. Parasitol. 41, 1129-1137.

Liu, Q., Zhang, C., Sun, A., Zheng, Y., Wang, L., and Cao, X. (2009). Tumor-educated CD1lbhighIalow regulatory dendritic cells suppress $\mathrm{T}$ cell response through arginase I. J. Immunol. 182, 6207-6216.

Lukacs-Kornek, V., Malhotra, D., Fletcher, A. L., Acton, S. E., Elpek, K. G., Tayalia, P., Collier, A. R., and Turley, S. J. (2011). Regulated release of nitric oxide by nonhematopoietic stroma controls expansion of the activated T cell pool in lymph nodes. Nat. Immunol. 12, 1096-1104.

Lutz, M. B. (2012). Therapeutic potential of semi-mature dendritic cells for tolerance induction. Front. Immunol. 3:123. doi:10.3389/fimmu.2012.00123

Malhotra, D., Fletcher, A. L., Astarita, J., Lukacs-Kornek, V., Tayalia, P., Gonzalez, S. F., Elpek, K. G., Chang, S. K., Knoblich, K., Hemler, M. E., Brenner, M. B., Carroll, M. C., Mooney, D. J., Turley, S. J., and Immunological Genome Project Consortium. (2012). Transcriptional profiling of stroma from inflamed and resting lymph nodes defines immunological hallmarks. Nat. Immunol. 13, 499-510.

Maroof, A., and Kaye, P. M. (2008). Temporal regulation of interleukin12p70 (IL-12p70) and IL-12-related cytokines in splenic dendritic cell subsets during Leishmania donovani infection. Infect. Immun. 76, 239-249.
Meredith, M. M., Liu, K., Darrasse-Jeze, G., Kamphorst, A. O., Schreiber, H. A., Guermonprez, P., Idoyaga, J., Cheong, C., Yao, K. H., Niec, R. E., and Nussenzweig, M. C. (2012). Expression of the zinc finger transcription factor zDC (Zbtb46, Btbd4) defines the classical dendritic cell lineage. J. Exp. Med. 209, 1153-1165.

Nguyen Hoang, A. T., Liu, H., Juaréz, J., Aziz, N., Kaye, P. M., and Svensson, M. (2010). Stromal cell-derived CXCL12 and CCL8 cooperate to support increased development of regulatory dendritic cells following Leishmania infection. J. Immunol. 185, 2360-2371.

Owens, B. M. J., Beattie, L., Moore, J. W. J., Brown, N., Mann, J. L., Dalton, J. E., Maroof, A., and Kaye, P. M. (2012). IL-10 producing Th1 cells and disease progression are regulated by distinct $\mathrm{CD} 11 \mathrm{c}+$ cell populations during visceral leishmaniasis. PLoS Pathog. 8, el002827. doi:10.1371/journal.ppat.1002827

Provenzano, P. P., Cuevas, C., Chang, A. E., Goel, V. K., Von Hoff, D. D., and Hingorani, S. R. (2012). Enzymatic targeting of the stroma ablates physical barriers to treatment of pancreatic ductal adenocarcinoma. Cancer Cell 21, 418-429.

Qian, C., An, H., Yu, Y., Liu, S., and Cao, X. (2007). TLR agonists induce regulatory dendritic cells to recruit Th1 cells via preferential IP-10 secretion and inhibit Th1 proliferation. Blood 109, 3308-3315.

Qian, C., Jiang, X., An, H., Yu, Y., Guo, Z., Liu, S., Xu, H., and Cao, X. (2006). TLR agonists promote ERK-mediated preferential IL-10 production of regulatory dendritic cells (diffDCs), leading to NK-cell activation. Blood 108, 2307-2315. 
Saraiva, M., and O'Garra, A. (2010). The regulation of IL-10 production by immune cells. Nat. Rev. Immunol. 10, 170-181.

Satpathy, A. T., Kc, W., Albring, J. C., Edelson, B. T., Kretzer, N. M., Bhattacharya, D., Murphy, T. L., and Murphy, K. M. (2012). Zbtb46 expression distinguishes classical dendritic cells and their committed progenitors from other immune lineages. J. Exp. Med. 209, 1135-1152.

Siegert, S., Huang, H. Y., Yang, C. Y., Scarpellino, L., Carrie, L., Essex, S., Nelson, P. J., Heikenwalder, M., Acha-Orbea, H., Buckley, C. D., Marsland, B. J., Zehn, D., and Luther, S. A. (2011). Fibroblastic reticular cells from lymph nodes attenuate $\mathrm{T}$ cell expansion by producing nitric oxide. PLoS ONE 6, e27618. doi:10.1371/journal.pone. 0027618

Smith, K. A., Hochweller, K., Hämmerling, G. J., Boon, L., MacDonald, A. S., and Maizels, R. M. (2011). Chronic helminth infection promotes immune regulation in vivo through dominance of
CD11cloCD103-dendritic cells. J. Xia, S., Guo, Z., Xu, X., Yi, H., Immunol. 186, 7098-7109.

Steinman, R. M., Hawiger, D., and Nussenzweig, M. C. (2003). Tolerogenic dendritic cells. Annu. Rev. Immunol. 21, 685-711.

Svensson, M., and Kaye, P. M. (2006). Stromal-cell regulation of dendriticcell differentiation and function. Trends Immunol. 27, 580-587.

Svensson, M., Maroof, A., Ato, M., and Kaye, P. M. (2004). Stromal cells direct local differentiation of regulatory dendritic cells. Immunity 21, 805-816.

Tang, H., Guo, Z., Zhang, M., Wang, J., Chen, G., and Cao, X. (2006). Endothelial stroma programs hematopoietic stem cells to differentiate into regulatory dendritic cells through IL-10. Blood 108, 1189-1197.

Wakkach, A., Fournier, N., Brun, V., Breittmayer, J. P., Cottrez, F., and Groux, H. (2003). Characterization of dendritic cells that induce tolerance and $\mathrm{T}$ regulatory 1 cell differentiation in vivo. Immunity 18 , 605-617. dendritic cells. Nat. Immunol. 5, 1124-1133.

Hepatic microenvironment programs hematopoietic progenitor differentiation into regulatory dendritic cells, maintaining liver tolerance. Blood 112, 3175-3185.

Xu, X., Yi, H., Guo, Z., Qian, C., Xia, S., Yao, Y., and Cao, X. (2012). Splenic stroma-educated regulatory dendritic cells induce apoptosis of activated CD4 T cells via Fas ligand-enhanced IFN-gamma and nitric oxide. J. Immunol. 188, 1168-1177.

Yasunaga, M., Manabe, S., Tarin, D., and Matsumura, Y. (2011). Cancerstroma targeting therapy by cytotoxic immunoconjugate bound to the collagen 4 network in the tumor tissue. Bioconjug. Chem. 22, 1776-1783.

Zhang, M., Tang, H., Guo, Z., An, H., Zhu, X., Song, W., Guo, J., Huang, X., Chen, T., Wang, J., and Cao, X. (2004). Splenic stroma drives mature dendritic cells to differentiate into regulatory
Conflict of Interest Statement: The authors declare that the research was conducted in the absence of any commercial or financial relationships that could be construed as a potential conflict of interest.

Received: 02 July 2012; accepted: 01 August 2012; published online: 21 August 2012.

Citation: Owens BMJ and Kaye PM (2012) Stromal cell induction of regulatory dendritic cells. Front. Immun. 3:262. doi: 10.3389/fimmu.2012.00262

This article was submitted to Frontiers in Antigen Presenting Cell Biology, a specialty of Frontiers in Immunology. Copyright (C) 2012 Owens and Kaye. This is an open-access article distributed under the terms of the Creative Commons Attribution License, which permits use, distribution and reproduction in other forums, provided the original authors and source are credited and subject to any copyright notices concerning any third-party graphics etc. 